\title{
Detecting and Correcting Instabilities in STEM Images
}

N. Braidy, ${ }^{1,2,5}$ Y. Le Bouar, ${ }^{2}$ S. Lazar, ${ }^{3,4}$ and C. Ricolleau ${ }^{5}$

${ }^{1}$ Chemical and Biotechnological Engineering, Université de Sherbrooke, 2500 Boul. Université Sherbrooke, PQ, Canada, J1K 2R1

${ }^{2}$ LEM, CNRS-ONERA, 29 Av. de la Division Leclerc, BP 72, 92322 Châtillon Cedex, France.

${ }^{3}$ FEI Electron Optics, 5600 KA Eindhoven. The Netherlands

${ }^{4}$ Canadian Centre for Electron Microscopy. 1280 Main St. West. Hamilton, ON, Canada, L8S 4M1.

${ }^{5}$ MPQ, CNRS-Université Denis Diderot, 4 rue Elsa Morante, CC 7021, 75205 Paris Cedex, France.

Atomically-resolved scanning probe images of crystalline specimen are now routinely obtained using TEM equipped with a spherical aberration probe corrector. Unlike their HREM counterparts, STEM images are intrinsically sensitive to environmental and instrumental temporal vibrations which translate to random displacements of the probe. If the frequency of the instabilities is the same order or lower to the scanning rate, then rows of pixel will incorrectly register the position of the object and translate to vertical streaks in the Fourier transform (FT) convoluted to each reflections. The horizontal and vertical components of this offset will be referred to as flagging and skipping, respectively. We demonstrate the possibility of detecting and mapping instabilities from the information held within the streaks and propose a method to suppress their contribution to the image.

The algorithm is based on geometrical phase method [1] and consists of mapping the phase of the streaks associated to two independent reflections. The components of the offset are then deduced from the two maps by solving a system of two linear equations. The flagging correction is first performed on each row by shifting the row by the prescribed amount using the Fourier-shift theorem. Skipping is then corrected by interpolating the intensity of the two closest rows to the actual region of the sample being registered by the pixel row.

The algorithm was implemented into a Digital Micrograph ${ }^{\mathrm{TM}}$ script [2] and tested on STEM images acquired with an aberration-corrected Titan (FEI) TEM operating at $300 \mathrm{kV}$. Fig. 1a shows a Ge $<011>$ crystal which exhibits practically no instability, a feature that is confirmed by the weakness of the streaks observed in the Hanning FT (HFT) (Fig. 1a, insets). Applying the "correction" (Fig. 1b) to such an image leaves it practically unchanged, except for a repression of the weak streak and the image being "smoother" (resulting from the interpolation operation of the skipping correction).

Fig. 2a shows the STEM image of a replica of disordered CoPt (1:1) nanoparticles (NPs) epitaxiallygrown on a $\mathrm{NaCl}$ substrate by sequential laser deposition of $\mathrm{Co}$ and $\mathrm{Pt}$ [3]. The instabilities are obvious both in the image and the HFT. The restored image (Fig. 2b) is free of such instabilities and exhibits the expected shape of the atoms. The horizontal and vertical components of the phase of the streak are mapped in Figs. 3a and 3b, respectively, using the 020 and 200 streaks. These figures translate into a random row offset of $11 \mathrm{pm}$, part of which is assigned to the intrinsic vibration associated to the interaction between an intense electron probe and the NP.

The restoration procedure is proposed as a pre-processing step to real-space STEM image processing methods for quantitative analysis and as a diagnostic tool to evaluate the stability of an instrument. 
References

[1] M.J. Hÿtch, E. Snoeck and R. Kilaas Ultramicroscopy (1998) 74131.

[2] Script available on request to the author. IPU (www.hremresearch.com) and Live Image Processing (www.felmi-zfe.tugraz.at/dm_scripts) packages must be installed.

[3] Y. Hirotsu and K. Sato. J. Ceramic Process. Res., (2005) 6236.

[4] Part of this work was done by NB as visiting scientist at the Canadian Centre for Electron Microscopy. We acknowledge Dr G. Botton for access to the facilities.
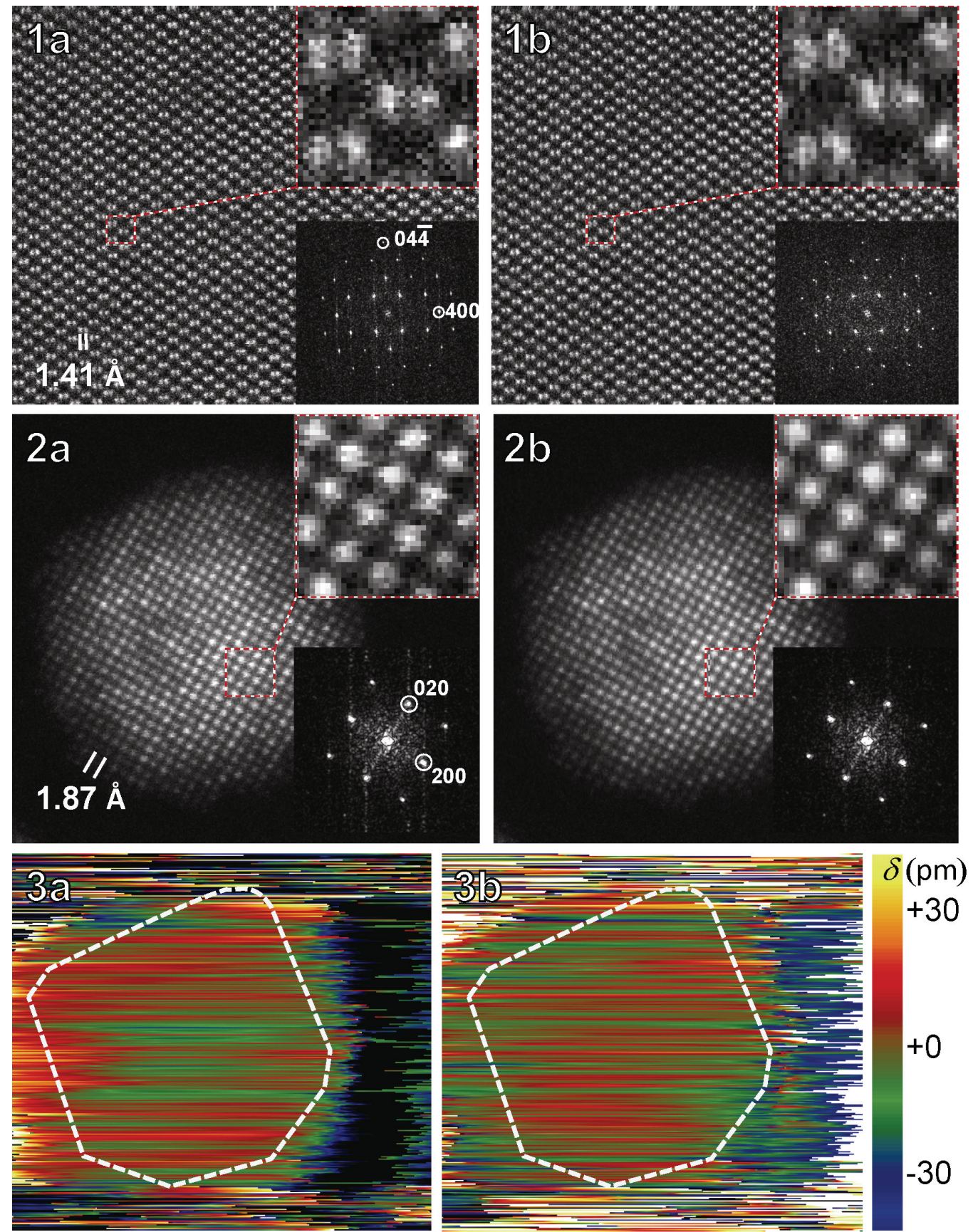

Unprocessed (Fig 1a) and processed (Fig. 1b) STEM image of a Ge $<011>$ crystal. Unprocessed (Fig 2a) and processed (Fig. 2b) STEM image of a CoPt NP. False color map of the flags (Fig. 3a) and the skips (Fig. 3b) with the outline (white dashed line) of the NP in Fig 2. 